\title{
ASSESSMENT OF NUTRITIONAL PARAMETERS OF NATIVE APPLE CULTIVARS AS NEW GENE SOURCES
}

\author{
J. Balík, O. Rop, J. Mlček, P. Híc, M. Horák, V. Řezníček
}

Received: April 6, 2012

\begin{abstract}
BALÍK, J., ROP, O., MLČEK, J., HÍC, P., HORÁK, M., ŘEZNÍČEK, V.: Assessment of nutritional parameters of native apple cultivars as new gene sources. Acta univ. agric. et silvic. Mendel. Brun., 2012, LX, No. 5, pp. 2738

Although there are tens of different apple varieties in Europe, only some of them were purposefully bred and selected in the past. In spite of the fact that they can have outstanding nutritional and technological properties, the majority of them are of only a local importance. The objective of this study was to show and popularize altogether 35 local apple varieties which are typical of the conditions of Central Europe. However, their genetic uniqueness represents an irreplaceable ecological wealth and for that reason these local varieties could become a new and outstanding source of nutrients and food. Today, they can be used not only for direct consumption and in food industry but also as a potential material for further breeding and selection. It was found out in our experiments that the highest content of total acids was shown by the variety 'Citronové zimní' (6.1 g. $\mathrm{kg}^{-1}$ of fresh matter). As far as the content of pectins was concerned, the highest levels were found out in the variety 'Strymka' (3.26\% in fresh matter). Of minerals, potassium showed the highest levels in fruit; for example, the variety 'Boikovo' contained $9.70 \mathrm{ppm}$ of this element in dry matter. Peels showed approximately 7 times higher antioxidant capacity than the flesh. For example, peels of the variety 'Bernské růžové' showed antioxidant capacity of as much as $56.65 \mathrm{mmol}$ of trolox per $\mathrm{kg}$ of fresh matter. This paper should contribute to efforts focused on saving of genetic variability of apples and their further application both in human nutrition and a further breeding and selection under conditions of pomological and alimentary practice.
\end{abstract}

apples, local varieties, titrable acidity, pectins, minerals, antioxidant capacity

Today, apple trees are the most popular and the most grown core fruit species in Europe. Due to their nutritional properties, apples are rather valued as fruit species (Gallus et al., 2005). Thanks to the content of minerals and polyphenolic compounds the consumption of apples is recommended to prevent cardiovascular or oncogenic diseases (Wojdylo et al., 2008).

In association with the process of intensification of agricultural production, the last century was characterised by extensive growing and breeding of only several selected and nowadays commercially successful apple varieties. Tens of others are only on the margin of general interest. A further propagation of local (native) cultivars is prevented above all by the fact that their sensory and technological properties are not as good as those of purposefully bred and selected ones although their local importance may be rather considerable (Goland and Bauer, 2004). Nevertheless, many of these native varieties show a significant tolerance to pathogens and pests, a high adaptability to less favourable climatic and soil conditions, good resistance to stress (Tetera, 2003) and the uniqueness of their relationship to nutritional properties (Toth et al., 2004).

In the Czech Republic, apple trees are the most frequent fruit species. The main objective of this paper is to emphasize some nutritional parameters of 35 typical Czech local apple varieties. During the process of landscape cultivation and care, 
many of the recommended apple varieties were spreading in Bohemia and Moravia in the past. This process was also accompanied with the selection of local apple varieties (Hričovský et al., 2003). The described varieties, however, could partly find a wider application in human nutrition (Monschein et al., 2006) and it is also necessary to mention the possibility of their application in the breeding work and selection (Laurens, 1996).

\section{MATERIAL AND METHODS}

The main hypothesis of this paper is not to prove the dependence existing between model factors on the one hand and nutritional parameters on the other. Its main objective is to emphasize the abundance of genetic diversity as related to growing and utilisation of apple trees above all due to the fact that the Czech Republic represents historically one of the richest breeding potentials in the world. For experiments, altogether 35 apple varieties were used; some of them, however, originated not only from Bohemia and Moravia but also from some other countries. In spite of this, the majority of them are typical just of the territory of Central Europe and it is not possible to neglect or ignore their, often hundred-year-old, use for nutritional purposes (Tetera, 2006). This paper compares nutritional parameters of individual varieties, tries to point out their prospects and to contribute to the preservation of their originality for the contemporary pomicultural practice. Its most important objective is to save the genetic variability of apple trees and to enable their further use in the fields of human nutrition and pomicultural practice.

\section{Sample collection and their preparation for chemical analyses}

Apples were harvested from five trees of each variety under study in the stage of consume ripeness. Each of these samples involved 3 replications from each tree (i.e. altogether 15). The samples were stored in a controlled environment at the temperature of +2 ${ }^{\circ} \mathrm{C}$ and under conditions of $85 \%$ of relative humidity (Kyzlink, 1990). For analyses all fruit without core were used (i.e. peel and flesh together). The samples were obtained from 35 apple tree varieties, 29 of which originated from the Tišnov region. The other samples were harvested in the White Carpathians in the cadastre of Valašské Klobouky. In the past the White Carpathians were declared as a protected landscape area and the described varieties are quite typical of this region (Tetera, 2006).

\section{Description of locality Tišnov}

Fruits were harvested in experimental orchards of Mendel University in Brno. These orchards are situated in the Tišnov vicinity, the Czech Republic. The average altitude is $435 \mathrm{~m}$ above sea level, and the mean annual temperature and precipitation are $7.5^{\circ} \mathrm{C}$ and $696 \mathrm{~mm}$, respectively. The soil type was classified as the Mesotrophic Cambisol.

\section{Description of locality Valašské Klobouky}

Fruits were harvested in experimental orchards of Tomas Bata University in Zlin. These orchards are situated in the south-western part of the White Carpathians near Zlín, the Czech Republic. The average altitude is $340 \mathrm{~m}$ above sea level, and the mean annual temperature and precipitation are $7.9^{\circ} \mathrm{C}$ and $760 \mathrm{~mm}$, respectively. The soil type was classified as the Mesotrophic Cambisol.

\section{Chemical analyses}

With the exception of the determination of pectin substances, vitamin $C$ and antioxidant capacity, all other chemical analyses were performed using the standard methods described by Novotný (2000).

Dry matter content was measured after drying off to a constant weight at the standard temperature of $105{ }^{\circ} \mathrm{C} \pm 2{ }^{\circ} \mathrm{C}$ - the apparatus VENTICELL 111 (BMT, Brno, Czech Republic). Soluble solid content (SSC) was estimated by means of polarimetric measurements in juice obtained after squeezing the fruit using a digital instrument HI 96801 (Hanna Instruments, Woonsocket, RI, USA). The content of total acids was measured by potentiometric titration; $20 \mathrm{~g}$ of homogenised sample were extracted for 30 minutes in a shaker in $200 \mathrm{ml}$ of re-distilled water at the temperature of $80^{\circ} \mathrm{C}$. The obtained extract was filtered and titrated with sodium hydroxide to the $\mathrm{pH}$ value of 8.1 by using the apparatus $\mathrm{pH} 211$ (Hanna Instruments, Woonsocket, RI, USA). The obtained result was converted to the content of acids in $\mathrm{g} . \mathrm{kg}^{-1}$ of fresh matter (FM).

\section{Mineral content assay}

The sample was dried off to a constant weight - the apparatus VENTICELL 111 (BMT, Brno, Czech Republic) - in a drier at the temperature of $105^{\circ} \mathrm{C} \pm 2{ }^{\circ} \mathrm{C}$. A portion of $\mathrm{lg}$ of homogenised dry matter (DM) (the size of particles up to $1 \mathrm{~mm}$ ) was thereafter mineralized in a mixture of concentrated sulphuric acid and 30\% hydrogen peroxide. Mineralized samples were quantitatively transferred into a $250 \mathrm{ml}$ volumetric flask and its volume was refilled to the volume with re-distilled water. The mineralizate was measured in an atomic absorption spectrometer PHILIPS PU 9200X (Philips, Amsterdam, the Netherlands). The content of total nitrogen was measured according to Kjeldahl using the apparatus KJELTEC TM 2300 (Foss, Hillerod, Denmark). The amount of minerals was expressed as ppm (respectively ppb) in DM.

\section{Pectin substances content assay}

The content of pectins was measured by means of a modified method described by Rop et al. (2008). A pulp sample ( $10 \mathrm{~g}$ ) was extracted at the temperature of $80{ }^{\circ} \mathrm{C}$ in a shaker with hydrochloric acid $\mathrm{c}=1 \mathrm{~mol} \cdot \mathrm{dm}^{-3}$ for a period of 90 minutes. The obtained hydrolyzate was quantitatively transferred into a $250 \mathrm{ml}$ volumetric flask and refilled to the volume with water. Pectins 
were thereafter measured photometrically as a coloured complex consisting of the product of thermal decomposition of galacturonic acid with $m$-hydroxybiphenyl in concentrated $\mathrm{H}_{2} \mathrm{SO}_{4}$. The samples of $5 \mathrm{ml}$ were gradually taken off and put into 50-ml flasks; thereafter, they were mixed with $6 \mathrm{ml}$ of sodium tetraborate $\left(\mathrm{c}=0.013 \mathrm{~mol} . \mathrm{dm}^{-3}\right)$ dissolved in concentrated sulphuric acid, filled up to the volume with distilled water and boiled for 5 minutes. Boiled samples were let to stand for 20 minutes and thereafter they were measured (at 520 $\mathrm{nm})$ together with standards in the apparatus LIBRA S6 (Biochrom Ltd., Cambridge, UK). The content of pectins was expressed in percents of fresh matter.

\section{Determination of ascorbic acid}

The determination of ascorbic acid content was carried out by a modified method of Wagner et al. (1979) and Miki (1981). $5 \mathrm{~g}$ of the sample were weighed in Erleymayer flask by adding $25 \mathrm{ml}$ of extractant methanol: $\mathrm{H}_{2} \mathrm{O}: \mathrm{H}_{3} \mathrm{PO}_{4}$ in the ratio 99:0.5:0.5. The flask with the samples was placed into a water bath with the temperature of $25^{\circ} \mathrm{C}$ where the samples were extracted for 15 minutes. To keep out the samples of daylight, the flask was covered with aluminium foil during the preparation. After the extraction the content of the bank was filtrated through paper Filtrapak No. 390. The filtrate prepared in this way before injection was diluted in ration of extractant and filtrated again through a membrane filter Nylon (0.45 $\mu \mathrm{m}$ Nylon filter disk). The instrument used for ascorbic acid analysis consisted of a solvent delivery pump (ESA Inc., Chelmsford, USA) - Model 582, guard cell (ESA Inc., Chelmsford, USA) - Model $5010 \mathrm{~A}$, working electrode potential $\mathrm{Kl}=600 \mathrm{mV}$, $\mathrm{K} 2=650 \mathrm{mV}$, chromatographic column - Model Supelcosil LC8 (150.0 x $4.6 \mathrm{~mm}$ ), $5 \mu \mathrm{m}$ particle size and an electrochemical detector (Coulochem III). Chromatographic conditions were constant: $30^{\circ} \mathrm{C}$, as a mobile phase methanol was used: $\mathrm{H}_{2} \mathrm{O}: \mathrm{H}_{3} \mathrm{PO}_{4}$ = 99:0.5:0.5, (filtrated through a filter Nylon, $0.2 \mu \mathrm{m}$ ), type of .elution was isocratic, the flow rate of the mobile phase was $1.1 \mathrm{ml} . \mathrm{min}^{-1}$, retention time 1.9$2.0 \mathrm{~min}$. The content of ascorbic acid was calculated as mg.100 $\mathrm{g}^{-1}$ of fresh matter.

\section{Antioxidant capacity assay}

The peel was separated from three parts of the fruit with the scalpel and cut to pieces. The flesh was cut out from all the apple length in a shape of 3 cylinders with a diameter of $2 \mathrm{~mm}$. The weight of $0.5 \mathrm{~g}$ was separated from the homogenised samples and immediately $3 \mathrm{ml}$ of $50 \%$ methanol were added. After 15 minutes of extraction centrifugation at 3,000 rpm was performed.

The antioxidant capacity was determined by photochemiluminescence method (PCL) using KIT ACL (400.803) and the instrument Photochem (Analytik Jena AG, Germany). Free radicals (superoxide anion radicals) were produced by optical excitation (irradiation) of a photosensitiser (dye). These radicals were partially eliminated from the sample by reaction with the antioxidants present in the sample. The remaining radicals cause the luminescence in the measuring cell, thereby allowing determination of the antioxidant capacity of the extract sample. The samples were diluted to give signals lying within the range of the calibration curve and within the linear range of the instrument. The total antioxidant capacity (TAC) of the fresh apple flesh or fresh aplle peel was quantified by comparison with the Trolox standard (Balík et al., 2008).

\section{Statistical evaluation}

All results were evaluated using the ANOVA variation statistics programme. Statistical data were calculated according to Snedecor and Cochran (1967) when using the statistical package Unistat, v. 5.1 and Office Excel ${ }^{\circledR}$ Microsoft.

\section{RESULTS}

When evaluating the overall average values with regard to individual apples, the highest weights of one fruit were recorded in the varieties 'Sikulské' (on average $236.3 \mathrm{~g}$ ) and 'Lebelovo' from the locality near Tišnov (200.7 g). Also in the other locality there were relatively high average weights of 'Lebelovo' apples. Higher weights were for example recorded also in the varieties 'Limburské', 'Krasokvět' or 'Croncelské' (Tab. I). The variety 'Sikulské' showed also the highest content of dry matter (16.51\%). More than $16 \%$ of dry matter was recorded also in the varieties 'Starkrimson', 'James Grieve' and 'Hvězdnatá reneta'. The variety 'Starkrimson' showed a high content of soluble solid content (16.10\% FM). In the variety 'Strýmka' this value was $15.68 \%$. More than $14 \%$ of soluble solid content in FM was shown also in the varieties 'Panenské české' and 'Citronové zimní' (Tab. I).

The titration acidity and the content of pectin substances were other important technological parameters estimated in altogether 35 samples of varieties under study. In this case there were considerable differences among individual varieties. As far as the total content of acids was concerned, the measured values ranged from 1.3 g. $\mathrm{kg}^{-1} \mathrm{FM}$ ('Coxova reneta') to 6.1 g.kg-1 FM ('Citronové zimní'). Differences were also found out in the contents of pectins. The lowest levels were found out in the varieties 'Lebelovo' from the Tišnov region (1.11\% FM) and 'Boikovo' (1.14\% FM). The varieties 'Croncelské' and 'Jeptiška' contained both 1.15\% FM while the highest one was found out in the variety 'Strýmka' (3.26\% FM).

Of mineral elements, the highest contents in dry matter were those of potassium and the variety 'Boikovo' contained as much as 9.70 ppm. High contents of potassium were also found in the varieties 'Jeptiška', 'Gustavovo', 'Grávštýnské' or 'Albrechtovo'. In these varieties there were also high contents of other macroelements, above all of 
I: Selected quality parameters of apple cultivars $(n=15)$

\begin{tabular}{|c|c|c|c|c|c|}
\hline Cultivar & $\begin{array}{c}\text { Fruit } \\
\text { weight (g) }\end{array}$ & $\begin{array}{c}\text { Dry } \\
\text { matter (\%) }\end{array}$ & $\begin{array}{c}\text { Soluble solid } \\
\text { content (\%) }\end{array}$ & $\begin{array}{c}\text { Titratable } \\
\text { acidity }\left(\mathrm{g} \cdot \mathrm{kg}^{-1}\right)\end{array}$ & $\begin{array}{c}\text { Pectins } \\
(\%)\end{array}$ \\
\hline Albrechtovo/VK* & $99.2 \pm 7.6^{* *}$ & $11.14 \pm 0.03$ & $13.50 \pm 0.12$ & $3.80 \pm 0.27$ & $2.01 \pm 0.04$ \\
\hline Baumannova reneta/Ti & $142.6 \pm 6.4$ & $12.81 \pm 0.02$ & $13.85 \pm 0.01$ & $4.28 \pm 0.28$ & $2.90 \pm 0.04$ \\
\hline Bernské růžové/VK & $80.5 \pm 5.1$ & $11.23 \pm 0.15$ & $11.70 \pm 0.18$ & $2.64 \pm 0.12$ & $2.19 \pm 0.01$ \\
\hline Bláhovo oranžové/Ti & $89.7 \pm 4.8$ & $15.04 \pm 0.05$ & $12.96 \pm 0.02$ & $2.10 \pm 0.52$ & $1.78 \pm 0.09$ \\
\hline Boikovo/Ti & $110.4 \pm 10.3$ & $17.23 \pm 0.03$ & $13.22 \pm 0.02$ & $1.93 \pm 0.13$ & $1.14 \pm 0.03$ \\
\hline Boskoopské/Ti & $136.5 \pm 9.7$ & $13.67 \pm 0.03$ & $12.51 \pm 0.01$ & $1.90 \pm 0.18$ & $2.45 \pm 0.08$ \\
\hline Citronové zimní/Ti & $154.1 \pm 8.2$ & $15.97 \pm 0.06$ & $11.14 \pm 0.01$ & $6.10 \pm 0.48$ & $1.50 \pm 0.05$ \\
\hline Coxova reneta / $\mathrm{Ti}$ & $90.7 \pm 7.6$ & $10.62 \pm 0.17$ & $14.35 \pm 0.02$ & $1.30 \pm 0.29$ & $2.12 \pm 0.16$ \\
\hline Croncelské/Ti & $168.3 \pm 10.4$ & $15.24 \pm 0.28$ & $14.22 \pm 0.02$ & $2.50 \pm 0.24$ & $1.15 \pm 0.04$ \\
\hline Gdánský hranáč/Ti & $75.0 \pm 3.9$ & $13.44 \pm 0.05$ & $11.16 \pm 0.01$ & $4.30 \pm 0.36$ & $2.87 \pm 0.51$ \\
\hline Grávštynské/Ti & $126.8 \pm 7.1$ & $10.83 \pm 0.05$ & $13.87 \pm 0.02$ & $2.35 \pm 0.13$ & $1.44 \pm 0.02$ \\
\hline Gustavovo/Ti & $99.3 \pm 8.8$ & $15.66 \pm 0.03$ & $12.54 \pm 0.02$ & $2.50 \pm 0.22$ & $1.90 \pm 0.17$ \\
\hline Hájkova muš. reneta/Ti & $124.2 \pm 14.5$ & $14.20 \pm 0.04$ & $11.00 \pm 0.01$ & $2.90 \pm 0.14$ & $1.93 \pm 0.08$ \\
\hline Hvězdnatá reneta/VK & $119.0 \pm 4.1$ & $16.35 \pm 0.03$ & $11.60 \pm 0.18$ & $3.60 \pm 0.29$ & $1.33 \pm 0.02$ \\
\hline Jadernička moravská/VK & $88.2 \pm 4.0$ & $7.57 \pm 0.13$ & $13.30 \pm 0.22$ & $1.50 \pm 0.27$ & $2.84 \pm 0.14$ \\
\hline James Grieve/Ti & $147.5 \pm 8.4$ & $16.22 \pm 0.02$ & $14.05 \pm 0.01$ & $1.68 \pm 0.15$ & $2.02 \pm 0.07$ \\
\hline Jeptiška/Ti & $155.7 \pm 7.4$ & $12.37 \pm 0.04$ & $11.20 \pm 0.01$ & $5.40 \pm 0.57$ & $1.15 \pm 0.05$ \\
\hline Kalvil bílý podzimní/Ti & $161.4 \pm 10.1$ & $12.32 \pm 0.02$ & $12.01 \pm 0.01$ & $5.00 \pm 0.50$ & $1.92 \pm 0.04$ \\
\hline Krasokvět/Ti & $170.1 \pm 18.8$ & $14.57 \pm 0.06$ & $13.89 \pm 0.02$ & $3.10 \pm 0.29$ & $1.85 \pm 0.11$ \\
\hline Krátkostopka králov./Ti & $169.1 \pm 7.0$ & $11.84 \pm 0.06$ & $11.80 \pm 0.22$ & $3.20 \pm 0.24$ & $2.80 \pm 0.09$ \\
\hline Lebelovo/VK & $165.0 \pm 17.1$ & $12.87 \pm 0.10$ & $11.60 \pm 0.08$ & $4.90 \pm 0.54$ & $1.59 \pm 0.12$ \\
\hline Lebelovo/Ti & $200.7 \pm 44.7$ & $13.10 \pm 0.03$ & $11.75 \pm 0.02$ & $3.30 \pm 0.36$ & $1.11 \pm 0.10$ \\
\hline Lecar/Ti & $100.5 \pm 6.2$ & $12.30 \pm 0.03$ & $13.61 \pm 0.01$ & $5.20 \pm 0.08$ & $2.56 \pm 0.03$ \\
\hline Limburské/Ti & $198.8 \pm 9.3$ & $10.39 \pm 0.02$ & $13.14 \pm 0.01$ & $1.50 \pm 0.29$ & $2.09 \pm 0.13$ \\
\hline Matčino/VK & $135.1 \pm 11.6$ & $12.42 \pm 1.00$ & $12.00 \pm 0.14$ & $1.60 \pm 0.20$ & $1.88 \pm 0.06$ \\
\hline Matčino/Ti & $115.4 \pm 5.8$ & $14.21 \pm 0.07$ & $12.15 \pm 0.01$ & $1.90 \pm 0.39$ & $2.01 \pm 0.11$ \\
\hline Ontario/Ti & $125.8 \pm 8.9$ & $14.89 \pm 0.03$ & $13.57 \pm 0.01$ & $1.80 \pm 0.08$ & $1.88 \pm 0.05$ \\
\hline Panenské české/Ti & $111.2 \pm 10.8$ & $11.84 \pm 0.03$ & $14.86 \pm 0.02$ & $3.10 \pm 0.14$ & $1.18 \pm 0.07$ \\
\hline Parména zlatá/Ti & $91.1 \pm 5.4$ & $16.13 \pm 0.02$ & $12.17 \pm 0.01$ & $3.00 \pm 0.28$ & $1.18 \pm 0.05$ \\
\hline Sikulské/Ti & $236.3 \pm 39.0$ & $16.51 \pm 0.02$ & $13.84 \pm 0.01$ & $2.20 \pm 0.23$ & $1.63 \pm 0.11$ \\
\hline Sláva světa/Ti & $153.0 \pm 8.4$ & $13.64 \pm 0.08$ & $11.05 \pm 0.01$ & $3.10 \pm 0.47$ & $1.87 \pm 0.05$ \\
\hline Spartan/Ti & $82.8 \pm 6.8$ & $13.95 \pm 0.01$ & $13.90 \pm 0.01$ & $2.28 \pm 0.32$ & $2.40 \pm 0.04$ \\
\hline Starkrimson/VK & $54.1 \pm 2.4$ & $16.35 \pm 0.01$ & $16.10 \pm 0.41$ & $1.70 \pm 0.24$ & $1.52 \pm 0.12$ \\
\hline Strýmka/Ti & $111.3 \pm 7.5$ & $11.58 \pm 0.08$ & $15.68 \pm 0.01$ & $1.80 \pm 0.29$ & $3.26 \pm 0.17$ \\
\hline Šarlatka boračská/Ti & $82.9 \pm 5.2$ & $14.51 \pm 0.03$ & $12.88 \pm 0.02$ & $1.90 \pm 0.24$ & $1.86 \pm 0.02$ \\
\hline Ušlechtilé žluté/Ti & $95.5 \pm 5.9$ & $15.33 \pm 0.02$ & $11.85 \pm 0.02$ & $4.00 \pm 0.37$ & $1.58 \pm 0.06$ \\
\hline Vilémovo/VK & $134.7 \pm 5.7$ & $11.84 \pm 0.05$ & $11.40 \pm 0.08$ & $2.60 \pm 0.14$ & $2.02 \pm 0.10$ \\
\hline Vilémovo/Ti & $123.5 \pm 6.9$ & $15.64 \pm 0.04$ & $12.15 \pm 0.02$ & $3.50 \pm 0.32$ & $2.55 \pm 0.05$ \\
\hline
\end{tabular}

*VK = Valašské Klobouky, Ti = Tišnov; **mean \pm standard deviation $(\mathrm{n}=15)$

nitrogen (Tab. II). So, for example, a high content of nitrogen was found out in the variety 'Boskoopské' (4.50 ppm). The highest levels of phosphorus (1.07 ppm) were found out in the varieties 'Bláhovo oranžové' and 'Jadernička moravská'. As far as the other elements were concerned, the content e.g. of calcium ranged from $0.35 \mathrm{ppm}$ (the variety 'Vilémovo') to $0.82 \mathrm{ppm}$ (the varieties 'Boikovo' and 'Gustavovo'). In case of magnesium this range had limits of $0.23 \mathrm{ppm}$ (the variety 'Bernské růžové') and
$0.41 \mathrm{ppm}$ (the varieties 'Croncelské' and 'Jeptiška') while in case of sodium the values ranged from 48 ppb (the variety 'Sláva světa') to 190 ppb (the variety 'Krátkostopka královská').

In the varieties harvested in the locality Valašské Klobouky the contents of vitamin $\mathrm{C}$ and of total antioxidant capacity were measured as well. While in the flesh the values of total antioxidant capacity ranged from 4.02 (the variety 'Vilémovo') to $6.59 \mathrm{mmol}$ of trolox. $\mathrm{kg}^{-1} \mathrm{FM}$ (the variety 'Bernské 
II: Content of elements in apple cultivars $(n=15)$

\begin{tabular}{|c|c|c|c|c|c|c|}
\hline \multirow{2}{*}{ Cultivar } & $\mathbf{N}$ & $\mathbf{P}$ & $\mathbf{K}$ & $\mathrm{Ca}$ & Mg & $\mathbf{N a}$ \\
\hline & (ppm) & (ppm) & (ppm) & (ppm) & (ppm) & (ppb) \\
\hline Albrechtovo/VK* & $3.68 \pm 0.15$ & $1.05 \pm 0.06$ & $9.10 \pm 0.14$ & $0.52 \pm 0.01$ & $0.34 \pm 0.04$ & $65 \pm 17$ \\
\hline Baumannova reneta/Ti & $3.90 \pm 0.14$ & $1.05 \pm 0.03$ & $7.10 \pm 0.22$ & $0.69 \pm 0.05$ & $0.34 \pm 0.01$ & $70 \pm 8$ \\
\hline Bernské růžové/VK & $3.08 \pm 0.10$ & $0.87 \pm 0.08$ & $6.30 \pm 0.29$ & $0.50 \pm 0.01$ & $0.23 \pm 0.03$ & $60 \pm 8$ \\
\hline Bláhovo oranžové/Ti & $3.83 \pm 0.22$ & $1.07 \pm 0.04$ & $7.70 \pm 0.35$ & $0.70 \pm 0.04$ & $0.39 \pm 0.03$ & $100 \pm 8$ \\
\hline Boikovo/Ti & $4.00 \pm 0.08$ & $0.97 \pm 0.02$ & $9.70 \pm 0.22$ & $0.82 \pm 0.01$ & $0.40 \pm 0.01$ & $103 \pm 10$ \\
\hline Boskoopské/Ti & $4.50 \pm 0.12$ & $0.99 \pm 0.06$ & $8.40 \pm 0.27$ & $0.58 \pm 0.03$ & $0.37 \pm 0.01$ & $90 \pm 12$ \\
\hline Citronové zimní/Ti & $3.20 \pm 0.18$ & $0.81 \pm 0.02$ & $7.10 \pm 0.29$ & $0.55 \pm 0.01$ & $0.39 \pm 0.03$ & $88 \pm 10$ \\
\hline Coxova reneta / Ti & $3.40 \pm 0.08$ & $1.04 \pm 0.04$ & $6.53 \pm 0.05$ & $0.51 \pm 0.04$ & $0.32 \pm 0.01$ & $70 \pm 8$ \\
\hline Croncelské/Ti & $3.73 \pm 0.54$ & $0.88 \pm 0.05$ & $8.50 \pm 0.18$ & $0.70 \pm 0.12$ & $0.41 \pm 0.02$ & $98 \pm 10$ \\
\hline Gdánský hranáč/Ti & $2.90 \pm 0.24$ & $0.85 \pm 0.04$ & $6.70 \pm 0.22$ & $0.39 \pm 0.01$ & $0.29 \pm 0.02$ & $80 \pm 8$ \\
\hline Grávštýnské/Ti & $4.00 \pm 0.08$ & $0.86 \pm 0.08$ & $9.20 \pm 0.18$ & $0.40 \pm 0.03$ & $0.28 \pm 0.01$ & $60 \pm 8$ \\
\hline Gustavovo/Ti & $3.28 \pm 0.21$ & $0.81 \pm 0.04$ & $9.00 \pm 0.43$ & $0.82 \pm 0.02$ & $0.32 \pm 0.01$ & $60 \pm 8$ \\
\hline Hájkova muš. reneta/Ti & $2.90 \pm 0.34$ & $0.93 \pm 0.08$ & $6.90 \pm 0.14$ & $0.59 \pm 0.05$ & $0.32 \pm 0.01$ & $90 \pm 8$ \\
\hline Hvězdnatá reneta/VK & $3.10 \pm 0.08$ & $0.87 \pm 0.02$ & $8.50 \pm 0.36$ & $0.48 \pm 0.02$ & $0.36 \pm 0.01$ & $68 \pm 10$ \\
\hline Jadernička moravská/VK & $3.20 \pm 0.14$ & $1.07 \pm 0.04$ & $7.80 \pm 0.29$ & $0.48 \pm 0.03$ & $0.30 \pm 0.01$ & $58 \pm 10$ \\
\hline James Grieve/Ti & $3.93 \pm 0.10$ & $1.04 \pm 0.04$ & $8.10 \pm 0.18$ & $0.64 \pm 0.05$ & $0.40 \pm 0.02$ & $135 \pm 13$ \\
\hline Jeptiška/Ti & $3.80 \pm 0.18$ & $0.86 \pm 0.02$ & $9.50 \pm 0.32$ & $0.80 \pm 0.08$ & $0.41 \pm 0.01$ & $90 \pm 8$ \\
\hline Kalvil bílý podzimní/Ti & $3.00 \pm 0.16$ & $0.88 \pm 0.05$ & $9.00 \pm 0.18$ & $0.51 \pm 0.03$ & $0.30 \pm 0.02$ & $60 \pm 8$ \\
\hline Krasokvět/Ti & $3.10 \pm 0.14$ & $1.00 \pm 0.04$ & $5.80 \pm 0.32$ & $0.46 \pm 0.01$ & $0.27 \pm 0.02$ & $138 \pm 5$ \\
\hline Krátkostopka králov./Ti & $4.10 \pm 0.20$ & $0.95 \pm 0.04$ & $7.20 \pm 0.38$ & $0.39 \pm 0.01$ & $0.33 \pm 0.02$ & $190 \pm 14$ \\
\hline Lebelovo/VK & $3.30 \pm 0.14$ & $1.02 \pm 0.03$ & $8.10 \pm 0.22$ & $0.41 \pm 0.02$ & $0.35 \pm 0.02$ & $188 \pm 5$ \\
\hline Lebelovo/Ti & $3.50 \pm 0.22$ & $0.95 \pm 0.07$ & $7.80 \pm 0.29$ & $0.41 \pm 0.01$ & $0.34 \pm 0.01$ & $140 \pm 14$ \\
\hline Lecar/Ti & $2.80 \pm 0.18$ & $0.89 \pm 0.03$ & $5.83 \pm 0.05$ & $0.48 \pm 0.02$ & $0.30 \pm 0.02$ & $60 \pm 8$ \\
\hline Limburské/Ti & $4.20 \pm 0.24$ & $0.90 \pm 0.03$ & $7.50 \pm 0.33$ & $0.53 \pm 0.05$ & $0.30 \pm 0.02$ & $90 \pm 8$ \\
\hline Matčino/VK & $3.30 \pm 0.14$ & $1.01 \pm 0.05$ & $7.80 \pm 0.32$ & $0.67 \pm 0.01$ & $0.34 \pm 0.03$ & $188 \pm 10$ \\
\hline Matčino/Ti & $3.50 \pm 0.41$ & $0.98 \pm 0.03$ & $8.20 \pm 0.18$ & $0.75 \pm 0.02$ & $0.32 \pm 0.02$ & $150 \pm 8$ \\
\hline Ontario/Ti & $3.70 \pm 0.08$ & $0.98 \pm 0.03$ & $8.80 \pm 0.36$ & $0.68 \pm 0.02$ & $0.36 \pm 0.03$ & $170 \pm 12$ \\
\hline Panenské české/Ti & $4.00 \pm 0.26$ & $0.88 \pm 0.03$ & $8.10 \pm 0.48$ & $0.75 \pm 0.04$ & $0.35 \pm 0.03$ & $170 \pm 16$ \\
\hline Parména zlatá/Ti & $3.00 \pm 0.14$ & $0.94 \pm 0.03$ & $6.50 \pm 0.18$ & $0.47 \pm 0.03$ & $0.33 \pm 0.02$ & $68 \pm 13$ \\
\hline Sikulské/Ti & $3.00 \pm 0.08$ & $0.95 \pm 0.04$ & $6.10 \pm 0.29$ & $0.59 \pm 0.01$ & $0.33 \pm 0.01$ & $70 \pm 8$ \\
\hline Sláva světa/Ti & $2.80 \pm 0.24$ & $0.89 \pm 0.05$ & $6.00 \pm 0.32$ & $0.51 \pm 0.04$ & $0.32 \pm 0.02$ & $48 \pm 5$ \\
\hline Spartan/Ti & $3.90 \pm 0.14$ & $1.01 \pm 0.06$ & $8.80 \pm 0.53$ & $0.66 \pm 0.05$ & $0.31 \pm 0.01$ & $118 \pm 10$ \\
\hline Starkrimson/VK & $3.30 \pm 0.32$ & $0.98 \pm 0.05$ & $6.30 \pm 0.14$ & $0.54 \pm 0.05$ & $0.29 \pm 0.06$ & $90 \pm 8$ \\
\hline Strýmka/Ti & $3.70 \pm 0.59$ & $1.02 \pm 0.06$ & $9.20 \pm 0.32$ & $0.63 \pm 0.03$ & $0.30 \pm 0.02$ & $120 \pm 8$ \\
\hline Šarlatka boračská/Ti & $3.40 \pm 0.16$ & $0.90 \pm 0.04$ & $6.70 \pm 0.36$ & $0.58 \pm 0.01$ & $0.28 \pm 0.03$ & $70 \pm 8$ \\
\hline Ušlechtilé zluté/Ti & $3.00 \pm 0.32$ & $0.81 \pm 0.03$ & $7.20 \pm 0.92$ & $0.60 \pm 0.03$ & $0.33 \pm 0.02$ & $80 \pm 8$ \\
\hline Vilémovo/VK & $3.30 \pm 0.12$ & $0.87 \pm 0.05$ & $6.30 \pm 0.14$ & $0.35 \pm 0.01$ & $0.27 \pm 0.02$ & $65 \pm 13$ \\
\hline Vilémovo/Ti & $3.50 \pm 0.18$ & $0.90 \pm 0.12$ & $7.20 \pm 0.42$ & $0.45 \pm 0.02$ & $0.33 \pm 0.01$ & $70 \pm 8$ \\
\hline
\end{tabular}

*VK = Valašské Klobouky, Ti = Tišnov; ***mean \pm standard deviation ( $\mathrm{n}=15$ )

růžové'), in peels these values ranged from 19.11 (again the variety 'Vilémovo') to $56.65 \mathrm{mmol}$ of trolox. $\mathrm{kg}^{-1}$ FM (again the variety 'Bernské růžové'); the obtained results were statistically significant (Tab. III). Statistically significant minimum contents of vitamin C were found out in apples of the varieties 'Jadernička moravská' and 'Starkrimson' (Tab. V). On the other hand, statistically significant maximum contents of vitamin $\mathrm{C}$ were found out in apples of the variety 'Albrechtovo' (13.55 mg. $\mathrm{kg}^{-1} \mathrm{FM}$ ). When estimating correlations existing between the content of ascorbic acid and the total antioxidant capacity, the calculated correlation coefficients for the flesh and peel were $r=0.7806$ and $r=0.4995$, respectively (Fig. 6). 


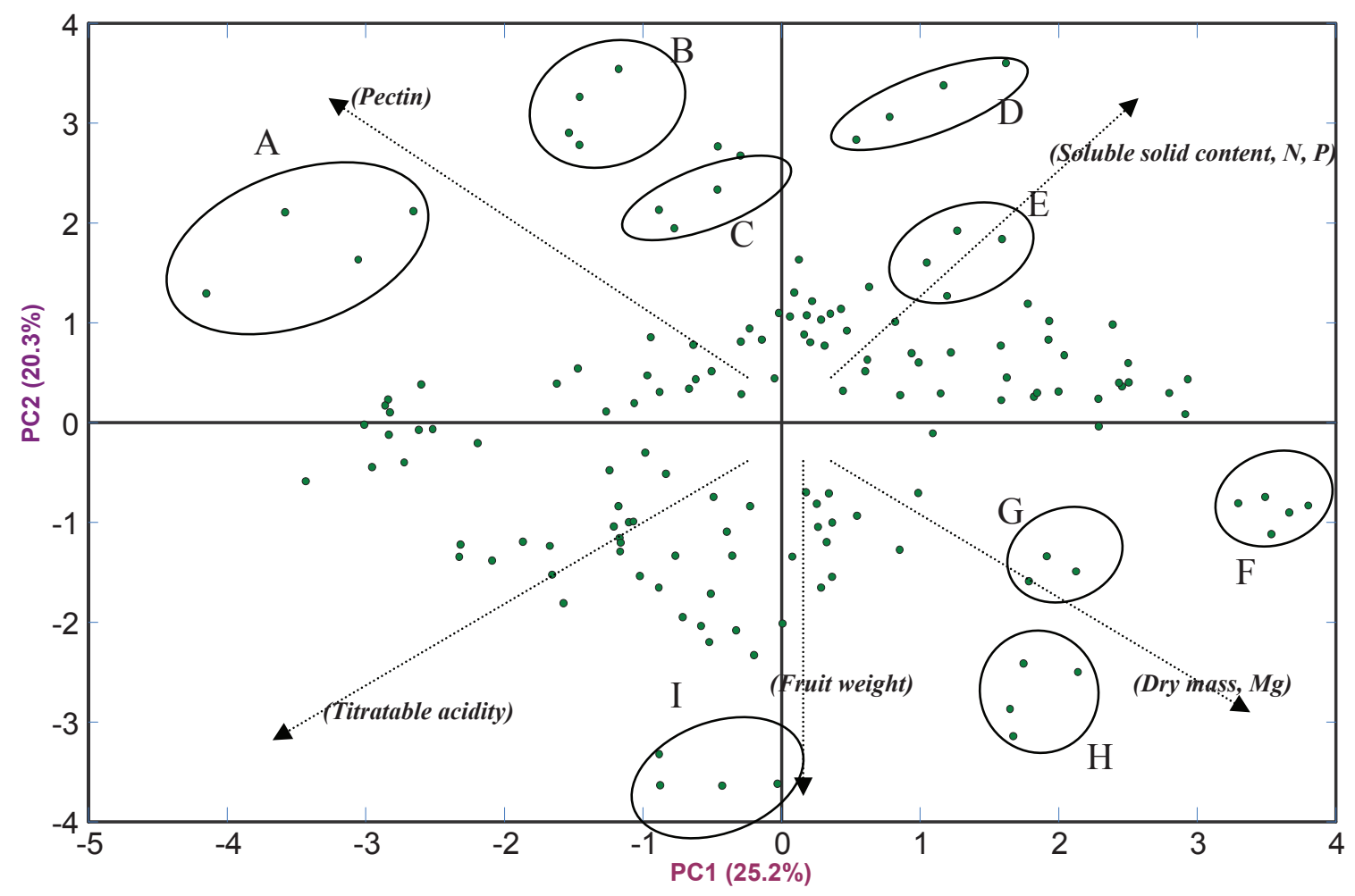

1: Principal component analysis of all observed analytic data

( $\mathrm{A}$ = Bernské růžové, $\mathrm{B}=$ Jadernička moravská, $\mathrm{C}=$ Coxova reneta, $\mathrm{D}=$ Strýmka, E = Spartan, F = Boikovo, G = Croncelské, H = Jeptiška, I = Citronové zimní)

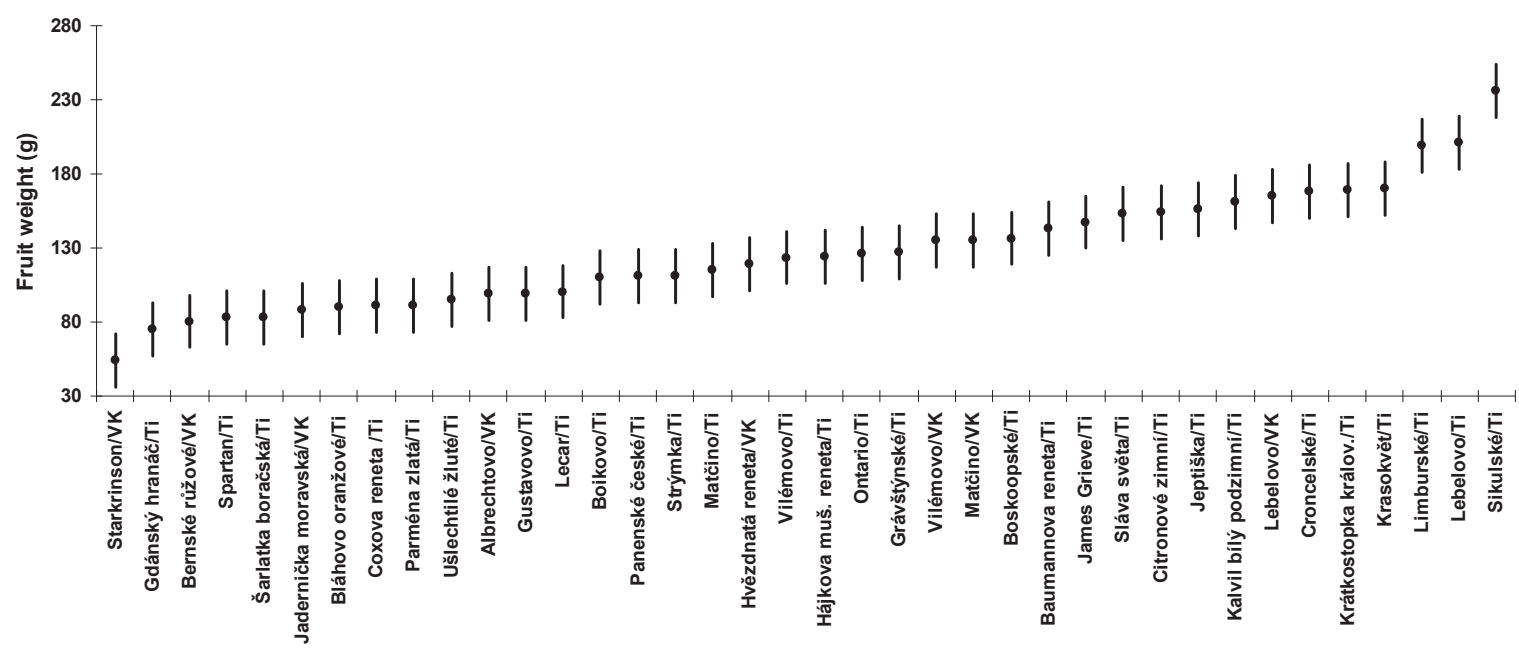

2: Mean of fruit weight and Tukey HSD intervals $(P=0.95 ; n=15)$ depending on apple cultivars

\section{DISCUSSION}

Today, apple trees are the most popular and the most grown core fruit species in Europe. As far as apples as fruit are concerned, their taste and their possible utilization for nutritional purposes represent the most valued characteristics. However, all properties of fruit which influence marketability of apples are of importance as well. What is also important is their capability to tolerate transport and storage conditions (Goland and Bauer, 2004).
Although it is possible to find out tens of various apple tree varieties in Europe, only some of them were purposefully bred and selected in the past (Radicato et al., 1995) and the same concerns also for example pears (Benitez, 1998). A further propagation of local varieties is prevented above all by the fact that their sensory and technological properties are not as good as those of purposefully bred and selected ones (Kuhn et al., 2003) although their local importance may be rather considerable 


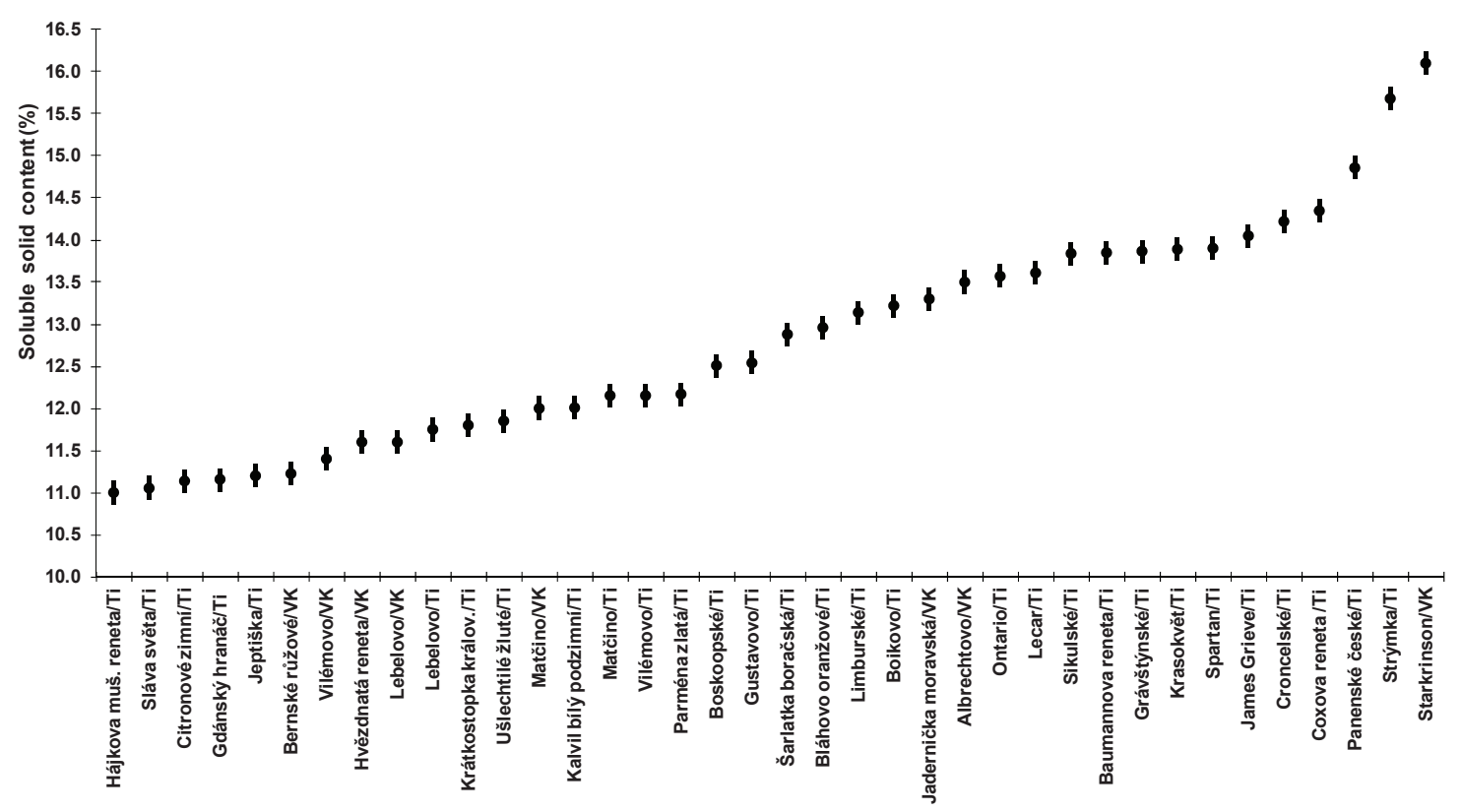

3: Mean of soluble solid content and Tukey HSD intervals $(P=0.95 ; n=15)$ depending on apple cultivars

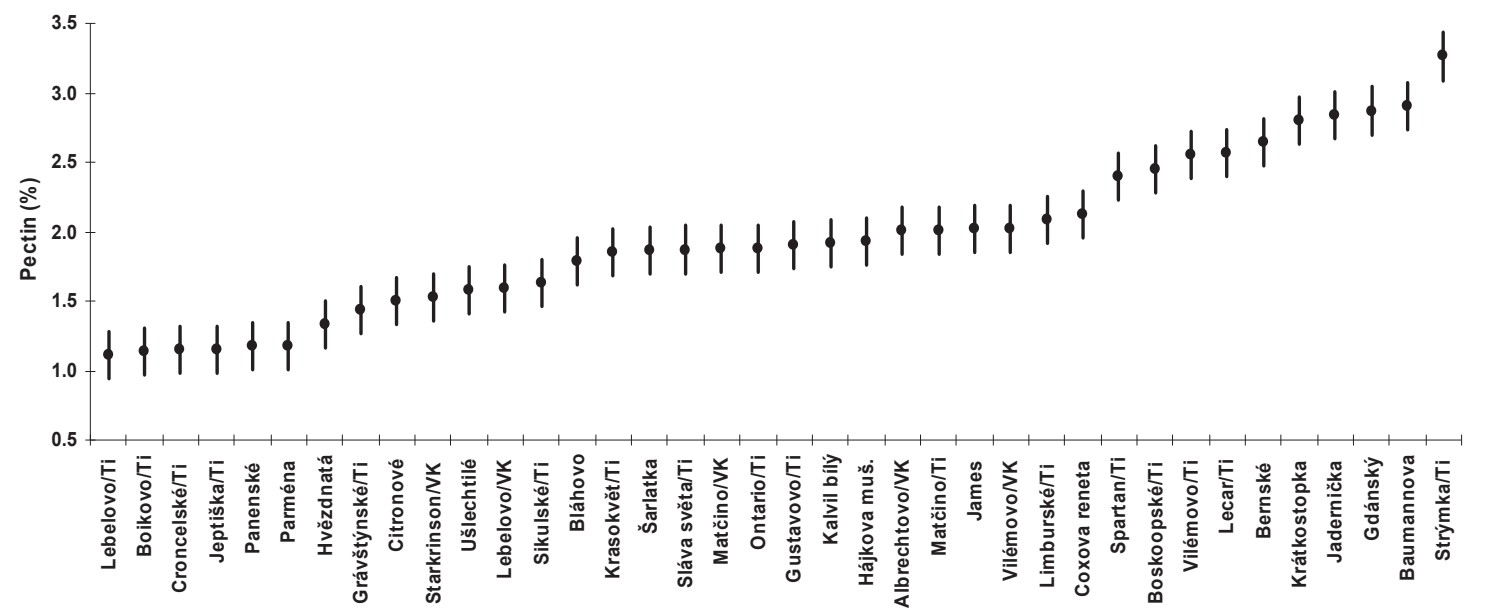

4: Mean of pectin content and Tukey HSD intervals $(P=0.95 ; n=15)$ depending on apple cultivars

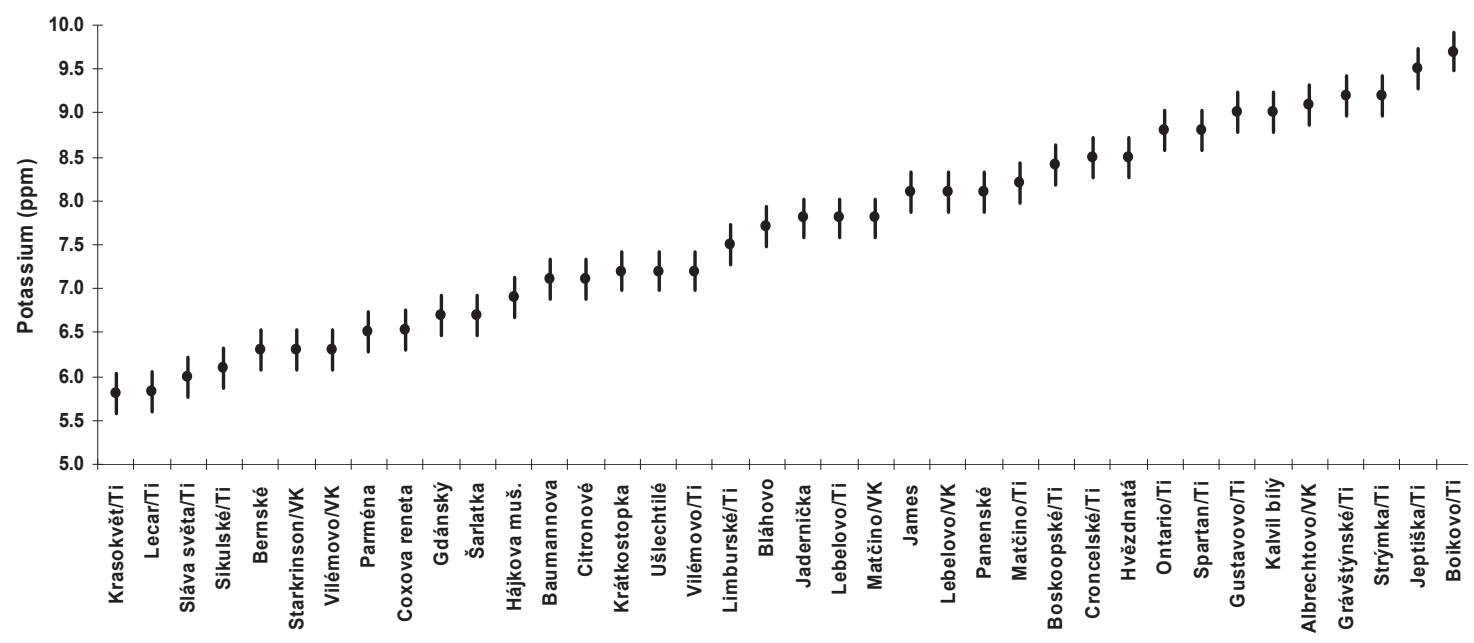

5: Mean of potassium content and Tukey HSD intervals $(P=0.95 ; n=15)$ depending on apple cultivars 
III: Antioxidant capacity in peel of selected apple cultivars

\begin{tabular}{|c|c|c|c|}
\hline \multirow{2}{*}{ Cultivar } & Mean & *HSD interval & \multirow{2}{*}{ Homogeneous groups } \\
\hline & \multicolumn{2}{|c|}{ (mmol trolox.kg-1 FM) } & \\
\hline Jadernička moravská & 19.11 & $10.16-28.03$ & A \\
\hline Lebelovo & 25.45 & $13.50-37.41$ & $\mathrm{AB}$ \\
\hline Vilémovo & 27.55 & $15.59-39.50$ & $\mathrm{AB}$ \\
\hline Starkrimson & 33.44 & $21.49-45.39$ & $\mathrm{ABC}$ \\
\hline Hvězdnatá reneta & 40.10 & $28.15-52.06$ & $\mathrm{BC}$ \\
\hline Albrechtovo & 41.73 & $29.78-53.69$ & $\mathrm{BC}$ \\
\hline Matčino & 46.55 & $34.60-58.51$ & $\mathrm{BC}$ \\
\hline Bernské růžové & 56.65 & $44.69-68.60$ & $\mathrm{C}$ \\
\hline Total mean & 36.32 & & \\
\hline
\end{tabular}

*high standard deviation (Tukey, $\mathrm{P}=0.95, \mathrm{n}=5$ )

IV: Antioxidant capacity in flesh of selected apple cultivars

\begin{tabular}{lccc}
\hline \multirow{2}{*}{ Cultivar } & Mean & *HSD interval & Homogeneous groups \\
\cline { 2 - 4 } & \multicolumn{2}{c}{ (mmol trolox.kg-1 FM) } & A \\
\hline Vilémovo & 4.02 & $0.70-7.33$ & A \\
Matčino & 4.20 & $0.88-7.51$ & $\mathrm{~A}$ \\
Starkrimson & 4.49 & $1.18-7.81$ & $\mathrm{~A}$ \\
Jadernička moravská & 4.82 & $1.51-8.13$ & $\mathrm{~A}$ \\
Hvězdnatá reneta & 5.73 & $2.42-9.05$ & $\mathrm{~A}$ \\
Albrechtovo & 6.00 & $2.68-9.31$ & $\mathrm{~A}$ \\
Lebelovo & 6.12 & $2.81-9.44$ & $\mathrm{~A}$ \\
Bernské růžové & 6.59 & $3.27-9.90$ & \\
\hline Total mean & 5.25 & & \\
\hline
\end{tabular}

*high standard deviation (Tukey, $\mathrm{P}=0.95, \mathrm{n}=5$ )

V: L-ascorbic acid content in selected apple cultivars $\left(m g .100 g^{-1}\right)$

\begin{tabular}{|c|c|c|c|}
\hline \multirow{2}{*}{ Cultivar } & Mean & *HSD interval & \multirow{2}{*}{ Homogeneous groups } \\
\hline & \multicolumn{2}{|c|}{ (mg.100 g $\left.\mathrm{g}^{-1} \mathrm{FM}\right)$} & \\
\hline Jadernička moravská & 9.14 & $8.83-9.45$ & A \\
\hline Starkrimson & 9.15 & $8.84-9.46$ & A \\
\hline Matčino & 10.36 & $10.05-10.67$ & B \\
\hline Lebelovo & 12.65 & $12.34-12.96$ & $\mathrm{D}$ \\
\hline Bernské růžové & 12.92 & $12.61-13.23$ & $\mathrm{D}$ \\
\hline Albrechtovo & 13.55 & $13.24-13.86$ & $\mathrm{E}$ \\
\hline Total mean & 11.43 & & \\
\hline
\end{tabular}

*high standard deviation (Tukey, $\mathrm{P}=0.95, \mathrm{n}=15$ )

(Goland and Bauer, 2004). Local varieties represent a rich source of genetically conditioned properties, i.e. not only their resistance to diseases but also some - until now - unknown and not described possibilities of a unique technological utilisation and specific taste and other sensory properties (Monschein et al., 2006). Although it is necessary to consider some differences in their chemical composition, resulting from the year of harvest and influenced by the locality (Little and Tailor, 1981), it can be said that local varieties are well adapted to local climatic conditions (Melounová et al., 2004). It is also known that there are only slight differences in the contents of individual chemical compounds when comparing individual varieties and that they are determined by the genetic uniqueness of each of them (Goland and Bauer, 2004).

In individual apple tree varieties the values of measured chemical characteristics ranged within the limits mentioned in tables used in alimentary industry (Kováčiková et al., 1997; Kopec, 1998). Nevertheless, individual varieties showed variability, 


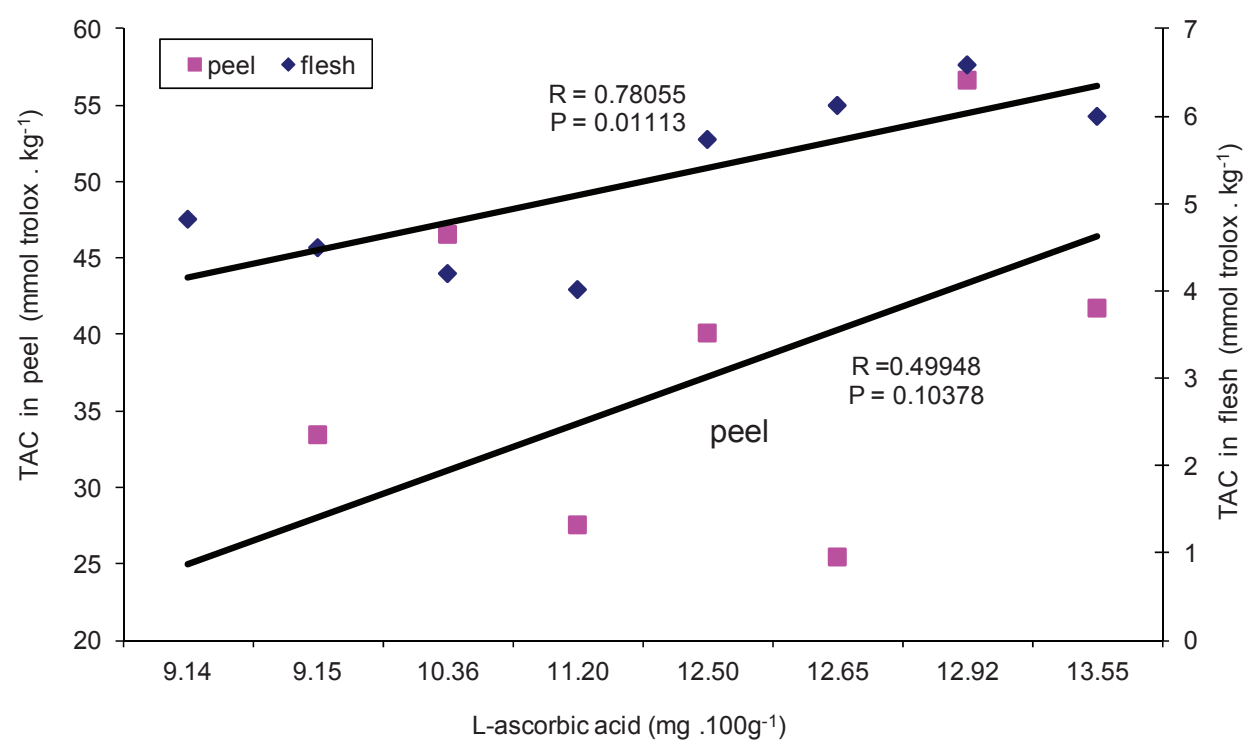

6: Correlation analysis between antioxidant capacity in peel (flesh) of apple cultivar and L-ascorbic content

which is presented in Tabs. I-V and Figs. 1-6. The similarity of chemical characteristics of individual apple varieties and their general affinity in the stage of consume ripeness is expressed by means of cluster analysis. When evaluating individual clusters it is obvious that the most marked characteristic is the kinship based on the stage of consume ripeness (Tetera, 2006).

Of chemical parameters under study, the most important are the following: the soluble solid content, the content of acids and the content of pectins (Rop et al., 2010a). In apples, the content of organic acids is represented mainly (90\%) by malic acid (Kyzlink, 1990). However, the presence of some macroelements (e.g. nitrogen) is manifested by an increase in the content of citric acid and a decrease in the malic acid (Jakopic et al., 2007). Among other organic acids it is possible to find out malonic, shikimic, and fumaric acids, some amino acids (with predominating aspartic acid) and polyphenolic chlorogenic acid (Stampar et al., 2002). So, for example, it was found out, similarly as Suni et al. (2000) noticed, that the content of acids in the variety 'Boskoopské' was low (1.9 g. $\mathrm{kg}^{-1}$ FM). On the other hand, the variety 'Citronové zimní' contained 6.1 g. $\mathrm{kg}^{-1}$ FM of organic acids. In the variety 'Boskoopské', the content of nitrogen in dry matter was also high (4.50 ppm of DM). Wolf et al. (2007) mentioned similar contents of nitrogen in apples (up to 5.00 ppm).

Apples are important above all due to their content of pectins, which predetermine them for the processing to fruit spreads thanks to their capability of gelification in presence of saccharose under conditions of low pH (Kyzlink, 1990). Of other core fruit species a high content of pectins can be found for example in quinces (Baker, 1997), which may contain as much as 3\% of pectins in FM (Kováčiková et al., 1997). In apples, however, the average content of pectins is about 1.1\% FM (Kopec, 1998). In our experiments, this value was found out in varieties with the lowest contents of pectin compounds (Tab. I). The variety 'Strýmka', which contained as much as $3.26 \%$ of pectins in FM, was an interesting exception. This variety is frequently used in the region of the White Carpathians (Tetera, 2006). However, this does not have to be an extreme value because Bailoni et al. (2005) mentioned that some local varieties may contain as much as $5 \%$ of pectins in FM.

As compared with other species of core fruit, apples show relatively high contents of mineral substances (Kovacs and Meresz, 2004). In core fruit, potassium is the most abundant macroelement (Kováčiková et al., 1997). In our experiments, the variety 'Boikovo' contained as much as $9.70 \mathrm{ppm}$ of this element in dry matter. Although some commercial varieties (e.g. 'Ontario' or 'Spartan') showed also high contents of macroelements, some typically local varieties contained more than $9.00 \mathrm{ppm}$ of potassium. Also the contents of other elements (phosphorus, magnesium, sodium) were relatively high in local varieties, even when compared with tabular values (Kováčiková et al., 1997; Kopec, 1998). Health benefits resulting from the consumption of apples due to their high content of minerals was emphasized also by several authors, e.g. by Biedrzycka (2008). It is well known that local varieties show very often higher contents of minerals than the commercial ones (Iwane, 1991).

The content of vitamin $\mathrm{C}$ in crops is most influenced by variety and year of cultivation Valšíková et al., 2010). The contents of vitamin C and antioxidant capacity were measured in apples showing the consume ripeness and originating from the locality Valašské Klobouky. As one can see in Tabs. III and IV, antioxidant capacity of peels was explicitly higher than of the flesh. This fact was 
mentioned also by Drogoudi et al. (2008) who found out that the peel can show as much as 9 times higher antioxidant capacity (in our experiments this was 7 times more). Authors Rop et al. (2010b) emphasized high values of antioxidant capacity of local varieties, which - as compared with commercial varieties - contained considerable amounts of phenolic substances. It was also found out that there were high correlations between antioxidant capacity and the content of phenolic substances (Gil et al. 2002). It was also found out that the correlation between antioxidant capacity and the content of ascorbic acid was also high (Schmitz-Eiberger et al., 2003). So, for example, for apples Thaipong et al. (2006) mentioned correlation coefficients ranging from $r=0.81$ to 0.97 . In our experiments the value of correlation coefficient was for the flesh and peel were $r=0.78$ and $r=0.49$, respectively (as compared with antioxidant capacity) (Fig. 6). In our measurement there was also a considerable variability of the contents of vitamin $\mathrm{C}$ in individual varieties (Tab. V) and it could be said that this was typical of apple trees (Tetera, 2006).

By analysis of main components original variables of observed parameters of fruit were substituted by main components (Fig. 1). The first two main components represented as axes in Fig. 1 explain on the whole $45.5 \%$ of all variability of original variables and divide apple cultivars to several clearly distinguishable groups (A, B, C, D, E, F, G, H and I). With the first main component parameters SSC, the contents of nitrogen, phosphorus, magnesium and dry matter correlated positively, but especially the contents of pectins and acids negatively. In particular the fruit weight correlated negatively with the second main component. Therefore, it is possible to, for example, claim that the fruit of the cultivar 'Jadernička moravská' belongs to the fruit with a lower weight and a significantly higher content of pectins, on the contrary, the fruits of the cultivar 'Citronové zimní' are representatives of the varieties with larger fruits and with higher contents of acids.

\section{SUMMARY}

This paper presents chemical characteristics of 35 local varieties of apple trees, which originated from two localities situated in the territory of the Czech Republic. All varieties were characterised by variability in the content of basic chemical components. From the technological and nutritional points of view some of them (e.g. pectins) are rather important and their highest content was found out in the variety 'Strýmka'. On the other hand, the variety 'Boikovo' showed a high content of mineral substances especially potassium (9.70 ppm in dry matter). It was found out in our experiments that the highest content of total acids was shown by the variety 'Citronové zimní' (6.1 $\mathrm{g} \cdot \mathrm{kg}^{-1}$ of fresh matter). Thanks to their antioxidant capacity, these local varieties can be used for prevention of many diseases. The antioxidant capacity of peels was as much as 7 times higher than that of the flesh. For example, peels of the variety 'Bernské růžové' showed antioxidant capacity of as much as $56.65 \mathrm{mmol}$ of trolox per kg of fresh matter. Many of the cultivars described in this study are not well known; practically, they were not described in literature and the research results presented here are unique, especially as far as their chemical composition is concerned. This paper should contribute to efforts focused on saving of genetic variability of apples and their further application both in human nutrition and a further breeding and selection under conditions of pomological and alimentary practice.

\section{REFERENCES}

BAILONI, L., SCHIAVON, S., PAGNIN, G., TAGLIAPIETRA, F., BONSEMBIANTE, M., 2005: Quanti-qualitative evaluation of pectins in the dietary fibre of 24 foods. Italian Journal of Animal Science. Vol. 4, No. 1, p. 49-58. ISSN 1828-051X.

BAKER, R. A., 1997: Reassessment of some fruit and vegetable pectin levels. Journal of Food Science. Vol. 62, No. 2, p. 225-229. ISSN 1750-3841.

BALÍK, J., KYSELÁKOVÁ, M., VRCHOTOVÁ, N., TŘÍSKA, J., KUMŠTA, M., VEVERKA, J., HÍC, P., TOTƯŠEK, J., LEFNEROVÁ, D., 2008: Relations between polyphenols content and antioxidant activity in vine grapes and leaves. Czech Journal of Food Science. Vol. 26, Special Issue, p. 25-32. ISSN 1212-1800.

BENITEZ, C., 1998: Harvesting and storing non traditional pear cultivars. VIIth International
Symposium on Pear Growing. Acta Horticulturae. Vol. 475, p. 535-542. ISSN 0567-7572.

BIEDRZYCKA, E., AMAROWICZ, R., 2008: Diet and health: Apple polyphenols as antioxidants. Food Reviews International. Vol. 24, No. 2, p. 235251. ISSN 8755-9129.

DROGOUDI, P. D., MICHAILIDIS, Z., PANTELIDISA, G., 2008: Peel and flesh antioxidant content and harvest quality characteristics of seven apple cultivars. Scientia Horticulturae. Vol. 115, No. 2, p. 149-153. ISSN 0304-4238.

GALLUS, S., TALAMINI, R., GIACOSA, A., MONTELLA, M., RAMAZZOTT,I V., FRANCESCHI, S., NEGR, IE., LA VECCHIA, C., 2005: Does an apple a day keep the oncologist away. Annals of Oncology. Vol. 16, No. 11, p. 1841844. ISSN 0923-7534. 
GIL, M. I., TOMAS-BARBERAN, F. A., HESSPIERCE, B., KADER, A. A., 2002: Antioxidant capacities, phenolic compounds, carotenoids, and vitamin $\mathrm{C}$ contents of nectarine, peach, and plum cultivars from California. Journal of Agricultural and Food Chemistry. Vol. 50, No. 17, p. 4976-4982. ISSN 0021-8561.

GOLAND, C., BAUER, S., 2004: When the apple falls close to the tree: Local food systeme and the preservation of diversity. Renewable Agriculture and Food Systeme. Vol. 19, No. 4, p. 228-236. ISSN 1742-1705.

HOPPNER, F., 2002: Speeding up Fuzzy c-Means: Using a Hierarchical Data Organisation to Control the Precision of Membership Calculation. Fuzzy Sets and Systems. Vol. 128, No. 3, p. 365-378. ISSN 0165-0114.

HRIČOVSKÝ, I., ŘEZNÍČEK, V., SUS, J., 2003: Jabloně, hrušně, kdouloně, mišpule, $1^{\text {st }}$ ed. Bratislava: Príroda, 104 p., ISBN 80-07-11223-5.

JAKOPIC, J., VEBERIC, R., ZUPANCIC, K., STAMPAR, F., 2007: Influence of nitrogen on the content of carbohydrates and organic acids in apples (Malus domestica Borkh.) cv. 'Golden Delicious'. European Journal of Horticultural Science. Vol. 72, No. 2, p. 66-72. ISSN 1611-4426.

IWANE, A., 1991: Effect of cultivars and year on mineral components of apples. Journal of the Japanese Society for Food Science and Technology. Vol. 38, No. 4, p. 329-336. ISSN 1341-027X.

KOPEC, K., 1998: Tabulky nutričních hodnot ovoce a zeleniny, $1^{\text {st }}$ ed. Praha: ÚZPI, 72 p., ISBN 8086153-64-9.

KOVACS, E., MERESZ, P., 2004: The effect of harvesting time on the biochemical and ultrastructural changes in Idared apple. Acta Alimentaria. Vol. 33, No. 3, p. 285-296. ISSN 01393006.

KOVÁČIKOVÁ, E., VOJTAŠŠÁKOVÁ, A., HOLČÍKOVÁ, K, 1997: Potravinové tabulky, $1^{\text {st }}$ ed. Bratislava: VÚP, 210 p., ISBN 80-85330-33-4.

KUHN, B. F., ANDERSEN, T. T., PEDERSEN, H. L., 2003: Evaluation of 14 old unsprayed apple varieties. Biological Agriculture \& Horticulture. Vol. 20, No. 4, p. 301-310. ISSN 0144-8765.

KYZLINK, V., 1990: Principles of Food Preservation, $1^{\text {st }}$ Ed. Amsterdam: Elsevier, 598 p., ISNB 0-44498844-0.

LAURENS, F., 1998: Review of the current apple breeding programmes in the world: Objectives for scion cultivar imrovement. Acta Horticulturae. Vol. 484, p. 163-170. ISSN 0567-7572.

LITTLE, C. R., TAYLOR, H. J., 1981: Orchard locality and storage factors affecting the commercial duality of Australian Granny Smith apples. Journal of Horticultural Science and Biotechnology. Vol. 56, No. 4, p. 323-329. ISSN 1462-0361.

MELOUNOVÁ, M., VEJL, P., SEDLÁK, P. REZNEROVÁ, A., TESAŘOVÁ, M., BLAŽEK, J., ZOUFALA, J., 2004: The variability of Ventura inaequalis CKE. races in the Czech Republic and the accumulation of resistence genes in apple germplasm. Plant Soil and Environment. Vol. 50, No. 9, p. 416-423. ISSN 1214-1178.

MIKI, N., 1981: High-performance liquidchromatographic determination of ascorbic acid in tomato products. Journal of the Japanese Society for Food Science and Technology. Vol. 28, No. 5, p. 264-268. ISSN 1341-027X.

MONSCHEIN, S., GRUB,E M., GRILL, D., 2006: Assessment of the genetic diversity of native apple cultivars in the south eastern ranges of the Alps with three selected microsatellite loci. Journal of Applied Botany and Food Quality-Angewandte Botanik. Vol. 80, No. 2, p. 135-137. ISSN 16139216.

NOVOTNÝ, F., 2000: Metodiky chemických rozborů pro hodnocení kvality odrůd, ${ }^{\text {st }}$ ed. Brno: ÚKZÚZ, 555 p., ISBN 80-86051-76-5.

RADICATTI, L., MARTINO, I., VERGANO, G., 1995: Apple and pear cultivars of Piemonte (Italy) in pomological descriptions of 18th and 19th centuries. Acta Horticulturae. Vol. 391, p. 273282. ISSN 0567-7572.

ROP, O., KRAMÁŘOVÁ, D., VALÁŠEK, P., BŘEZINA, P., 2008: Content of pectin in regional varieties of apples. Chemické Listy. Vol. 102, No. 9, p. 851. ISSN 1213-7103.

ROP, O., ŘEZNÍČEK, V., VALŠÍKOVÁ, M., JURÍKOVÁ, T., MLČEK, J., KRAMÁŘOVÁ, D., 2010a: Antioxidant Properties of European Cranberrybush Fruit (Viburnum opulus var. edule). Molecules. Vol. 15, No. 6, p. 4467-4477. ISSN 1420-3049.

ROP, O., MLČEK, J., JURÍKOVÁ, T., VALŠÍKOVÁ, M., SOCHOR, J., ŘEZNÍČEK, V., KRAMÁŘOVÁ, D., 2010b: Phenolic content, antioxidant capacity, radical oxygen species scavenging and lipid peroxidation inhibiting activities of extracts of five black chokeberry (Aronia melanocarpa (Michx.) Elliot) cultivars. Journal of Medicinal Plants Research. Vol. 4, No. 22, p. 2431-2437. ISSN 19960875.

SCHMITZ-EIBERGER, M., WEBER, V., TREUTTER, D., BAAB, G., LORENZ, J., 2003: Bioactive components in fruits from different apple varieties. Journal of Applied Botany-Angewandte Botanik. Vol. 77, No. 5-6, p. 167-171. ISSN 16139216.

SNEDECOR, G. W., COCHRAN, W. G., 1967: Statistical Methods, $6^{\text {th }}$ ed. Iowa State University Press, Iowa, $875 \mathrm{p}$.

STAMPAR, F., VEBERIC, R., ZADRAVEC, P., HUDINA, M., USENIK, V., SOLAR, A., OSTERC, G., 2002: Yield and fruit quality of apples cv. 'Jonagold' under hail protection nets. Gartenbauwissenschaft. Vol. 67, No. 5, p. 205-210. ISSN 0016-478X.

SUNI, M., NYMAN, M., ERIKSSON, N. A., BJORK, L., BJORCK, I., 2000: Carbohydrate composition and content of organic acids in fresh and stored apples. Journal of the Science of Food and Agriculture. Vol. 80, No. 10, p. 1538-1544. ISSN 1097-0010. 
TETERA, V., 2003: Záchrana starých a krajových odrůd ovocných dřevin, $1^{\text {st }}$ ed. Veselínad Moravou: ČSOP, 76 p., ISBN 80-903444-0-2.

TETERA, V., 2006: Ovoce Bílých Karpat, $1^{\text {st }}$ ed. Veselí nad Moravou: ČSOP, 310 p., ISBN 80-903444-5-3.

THAIPONG, K., BOONPRAKOB, U., CROSBY, K., CISNEROS-ZEVALLOS, L., BYRNE, D. H., 2006: Comparison of ABTS, DPPH, FRAP, and ORAC assays for estimating antioxidant activity from guava fruit extracts. Journal of Food Composition and Analysis. Vol. 19, No. 6-7, p. 669-675. ISSN 0889-1575.

TOTH, M., KASA, K., SZANI, Z. S., BALIKO, E., 2004: Traditional old apple cultivars as new gene sources for apple breeding. Acta Horticulturae. Vol. 663, p. 609-612. ISBN 0567-7572.

VALŠÍKOVÁ, M., ČERVENKA, J., BARKOCI, Š., SUDZINA, M., 2010: The evaluation of vitamin C content in fruits of vegetable pepper and tomato. Acta Universitatis Agriculturae et Silviculturae
Mendeleianae Brunensis. Vol. LVIII, No. 2, p. 281286. ISSN 0524-7463.

WAGNER, E. S., LINDLEY, B., COFFIN, R. D., 1979:

High-performance liquid-chromatographic determination of ascorbic acid in urine-effect on urinary-excretion profiles after oral and intravenous administration of vitamin C. Journal of Chromatography. Vol. 163, No. 2, p. 225-229. ISSN 0021-9673.

WAJDYLO, A., OSZMIANSKI, J., LASKOWSKI, P., 2008: Polyphenolic compounds and antioxidant activity of new and old apple varieties. Journal of Agricultural and Food Chemistry. Vol. 56, No. 15, p. 6520-6530. ISSN 0021-8561

WOLF, P., HABICH, A. C., BURKLE, M., KAMPHUES, J., 2007: Basic data on food intake, nutrient digestibility and energy requirements of lorikeets. Journal of Animal Physiology and Animal Nutrition. Vol. 91, No. 5-6, p. 282-288. ISSN 1439-0396.

Address

doc. Ing. Josef Balík, Ph.D., Ing. Pavel Híc, Ing. Miroslav Horák, Ústav posklizňové technologie zahradnických produktů, Mendelova univerzita v Brně, Valtická 337, 69144 Lednice, Česká republika, doc. Ing. Otakar Rop, Ph.D., Ing. Jiří Mlček, Ph.D., Ústav technologie a mikrobiologie potravin, Fakulta technologická, Univerzita Tomáše Bati ve Zlíně, Náměstí T. G. Masaryka 275, 76272 Zlín, Česká republika, prof. Ing. Vojtěch Řezníček, CSc., Ústav šlechtění a množení zahradnických rostlin, Mendelova univerzita v Brně, Valtická 337, 69144 Lednice, Česká republika, e-mail: balikj@zf.mendelu.cz, pavel.hic@mendelu.cz, miroslav.horak@mendelu. cz,rop@ft.utb.cz,mlcek@ft.utb.cz,vojtech.reznicek.usmzr@mendelu.cz 